\title{
Non-specific chronic low back pain: differences in spinal kinematics in subgroups during functional tasks
}

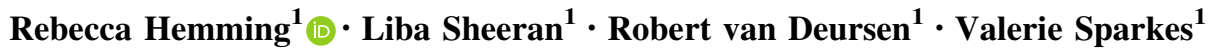

Received: 21 December 2016/Revised: 26 June 2017/ Accepted: 10 July 2017 / Published online: 21 July 2017

(c) The Author(s) 2017. This article is an open access publication

\begin{abstract}
Purpose A multidimensional classification approach suggests that motor control impairment subgroups exist in non-specific chronic low back pain (NSCLBP). Differences in sitting lumbar posture have been identified between two such subgroups [flexion pattern (FP) and active extension pattern (AEP)] and healthy individuals; however, functional spinal movement has not been explored. This study will evaluate whether NSCLBP subgroups exhibit regional spinal kinematic differences, compared to healthy individuals, during functional tasks.

Methods Observational, cross-sectional study design. Spinal kinematics of 50 NSCLBP subjects (27 FP, 23 AEP) and 28 healthy individuals were investigated using 3D motion analysis $\left(\right.$ Vicon $^{\mathrm{TM}}$ ) during functional tasks [reaching upwards, step down, step up, lifting, and replacing a box, stand-to-sit, sit-to-stand, bending to retrieve (and returning from retrieving) a pen from the floor]. Mean sagittal angle for the total thoracic, total lumbar, upper thoracic, lower thoracic, upper lumbar, and lower lumbar regions between groups was compared.

Results Significant differences were observed in lower thoracic and upper lumbar regions between NSCLBP subgroups during most tasks. Significant differences were
\end{abstract}

Electronic supplementary material The online version of this article (doi:10.1007/s00586-017-5217-1) contains supplementary material, which is available to authorized users.

Rebecca Hemming

HemmingRL@ cardiff.ac.uk

1 Arthritis Research UK Biomechanics and Bioengineering Centre, School of Healthcare Sciences, Cardiff University, Eastgate House, 35-43 Newport Road, Cardiff, Wales CF24 OAB, UK observed between the FP and healthy group in the lower thoracic region during stand-to-sit-to-stand tasks and bending (and returning from) to retrieve a pen from the floor. All significant results demonstrated the FP group to operate in comparatively greater flexion.

Conclusions The thoraco-lumbar spine discriminated between FP and AEP, and FP and healthy groups during functional tasks. FP individuals demonstrated more kyphotic thoraco-lumbar postures, which may be pain provocative. No significant differences were observed between AEP and healthy groups, suggesting that alternative mechanisms may occur in AEP.

Keywords Non-specific chronic low back pain . Functional movement $\cdot$ Kinematics · Lumbar · Thoracic

\section{Introduction}

Non-specific chronic low back pain (NSCLBP) is a complex heterogeneous biopsychosocial disorder with multiple manifestations [1]. Despite considerable NSCLBP research, there is little reported change in long-term prognosis [2] with intervention outcomes reported to be short term and moderate at best [3]. Difficulties in establishing effective interventions are likely to be due to an inability to define clear homogeneous NSCLBP subgroups; therefore, identifying specific NSCLBP subgroups using validated subclassification approaches is paramount [4].

For some NSCLBP individuals, the main driver for pain may be movement and posture behaviour indicating a mechanical basis for the disorder. A multidimensional classification system (MDCS), which considers such mechanisms, for example, maladaptive motor control 
impairment (MCI) (characterised by pain provocative behaviours), has been proposed [5].

This MDCS [5] outlines five NSCLBP MCI subgroups, where individuals are proposed to display full range of movement (ROM) in the direction of pain provocation and are clinically observed to habitually adopt end-range postures. These MCI subgroups are based upon subjective reporting of direction-specific aggravating and easing factors (described elsewhere [6]). MCI patients have been shown to display high levels of fear avoidance to adopt maladaptive end-range postures and movement strategies that may promote increased pain [6-8]. Treatment for these individuals focuses on reducing fear avoidance and optimising spinal control during functional activity to avoid end-range repetitive strain, reduce spinal loading, and subsequently reduce peripheral nociceptor sensitivity [5]. Previous work has established distinct physical characteristics between two proposed MCI subgroups (Active Extension Pattern and Flexion Pattern) and healthy individuals in adolescent and adult populations, with alterations in spinal position sense, spinal kinematics and trunk muscle activity observed during static postures [7-10].

Interventions focussed on targeted intervention for these MCI subgroups, such as Classification-based cognitive functional therapy (CB-CFT), have also been proposed to be effective when compared with usual care [11]. Despite CB-CFT incorporating functional re-education approaches, to date, no published work has evaluated how NSCLBP MCI subgroups operate during dynamic functional activities.

The purpose of this study is to better understand differences in spinal kinematics between NSCLBP subgroups and healthy individuals during functional tasks. This knowledge may assist in informing specific movement reeducation in CB-CFT and aid development of novel sensor and biofeedback technologies to promote back pain selfmanagement.

The hypothesis for this observational, cross-sectional study is that a difference in regional sagittal spinal curvatures between NSCLBP subgroups and healthy controls will be observed during a series of functional tasks.

\section{Materials and methods}

NSCLBP patients were recruited from routine physiotherapy waiting lists in Cardiff and Vale University Health Board (Cardiff, UK). Fifty NSCLBP and 28 healthy individuals volunteered. Ethical approval was obtained from The Research Ethics Committee 3 Wales (10/MRE09/28) within the Arthritis Research UK Biomechanics and Bioengineering Centre, Cardiff University. Informed consent was obtained from all participants. Sample size was based on lower lumbar sagittal spinal angle, which has been shown to discriminate between AEP and FP subgroups in sitting [7]. Power set at priori at 0.7 , alpha level of 0.05 , a sample of 24 subjects per group was calculated.

Inclusion and exclusion criteria are outlined in Table 1. NSCLBP subjects were classified independently by two physiotherapists (RH, LS) based on MDCS criteria [5]. Only subjects classified as FP or AEP (where both clinicians were in agreement) were included. To establish NSCLBP classification, a comprehensive subjective assessment including a full history of the individuals back pain, pain behaviour (including aggravating and easing postures and movements) and objective assessment was conducted. Full details of this procedure are published elsewhere [5, 12]. Gender, age, anthropometric data [weight, height, and body mass index (BMI)], and duration of pain were collected. Patient reported that measures for pain [Visual Analogue Scale (VAS)] [13], disability [Oswestry Disability Questionnaire (ODQ)] [14], distress [Distress and Risk Assessment Method (DRAM)] [15], and fear of movement [Tampa Scale of Kinesiophobia (TSK)] [16] were completed to evaluate group baseline characteristics. Data collection was conducted at the Research Centre for Clinical Kinesiology, Cardiff University, Wales, United Kingdom.

\section{Data collection}

Data were captured using an eight-camera Vicon ${ }^{\circledR}$ motion analysis system (Vicon 512 Motion Systems Ltd, Oxford, UK) at a sampling rate of 100 samples per second. Spherical retro-reflective markers $(10 \mathrm{~mm})$ (Vicon Motion Systems Ltd, Oxford, UK) were attached using doublesided tape over the following anatomical positions: spinous processes of the 7 th cervical, 2nd, 4th, 6th, 8th, 10th and 12th thoracic and 2nd and 4th lumbar vertebrae, manubrium sterni (superior border) and bilaterally on the anterior superior iliac spine (ASIS), posterior superior iliac spine (PSIS), iliac crest (mid-crest, vertically aligned with the greater trochanter) acromioclavicular joint, ulna styloid process, $10 \mathrm{~cm}$ lateral of the 12 th thoracic spinous process, lateral knee joint line, and the lateral malleoli creating a full body model (Fig. 1). The marker set used was customdesigned (Cardiff University, UK). To minimise, error cameras were calibrated prior to data collection and a chartered physiotherapist (RH) performed all marker placement [17].

Nine standardised functional tasks [reach up, sitting-tostanding, standing-to-sitting, step up, step down, box lift, box replace, bending to retrieve (and returning from retrieving) a pen from the floor] were evaluated. Tasks were chosen to reflect a range of usual functional activities and elicit a variation of flexion-related and extension- 
Table 1 Inclusion and exclusion criteria for the non-specific chronic low back pain (NSCLBP) group

\begin{tabular}{|c|c|}
\hline Inclusion criteria for the NSCLBP group & Exclusion criteria for the NSCLBP group \\
\hline $\begin{array}{l}\text { Aged 18-65 years } \\
\text { History of chronic LBP ( }>12 \text { weeks) } \\
\text { Pain in the lumbar and/or buttock region (defined as pain reported below } \\
\text { the level of T12 and no lower than the buttock line) } \\
\text { Clear mechanical basis of the disorder aligned with specific aggravating } \\
\text { and easing postures and movements, with distinct symptom relief } \\
\text { observed during movement conducted in the opposing direction of } \\
\text { reported pain provocation (assessed subjectively and objectively) } \\
\text { Clinical diagnosis of specific MCI-either FP or AEP }\end{array}$ & $\begin{array}{l}\text { Signs of serious spinal pathology (red flags) including significant } \\
\text { trauma, unexplained weight loss and widespread neurologic changes } \\
\text { Any vestibular, visual or neurological dysfunction affecting balance } \\
\text { Current radiating symptoms (and/or neurological deficit) below the } \\
\text { level of the buttock crease } \\
\text { Current pregnancy or breastfeeding } \\
\text { History of spinal surgery, fracture or malignancy } \\
\text { Inability to perform any of the functional tasks unaided } \\
\text { Inability to read written English language documents and follow } \\
\text { verbal instructions in English } \\
\text { Not fulfilling the inclusion criteria }\end{array}$ \\
\hline
\end{tabular}

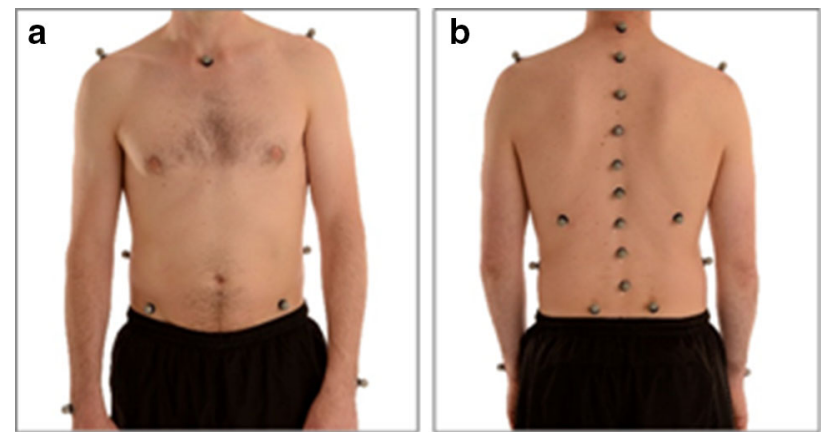

Fig. 1 Custom-designed marker set $\mathbf{a}$ anterior view and $\mathbf{b}$ posterior view

related tasks. Each task was repeated and recorded three times. Following each trial data was visualised in Vicon Nexus (Nexus 1.8.2 Vicon Motion Systems, Oxford, UK) to ensure that markers were consistently present (Fig. 2).

\section{Data processing and analysis}

Data processing was conducted in Vicon Nexus. The sacral marker S1 was calculated as the average point between PSIS markers. Processed trials were converted to a c3d file and run through a custom developed analysis programme in MATLAB (version R2013a, The Mathworks Inc., Natick, MA, USA). Prior to the calculation of spine curvatures, the coordinates of the motion capture markers on the spine were transformed from the global (lab) coordinate system to the local (pelvis) coordinate system. The spine was modeled as a curve in the sagittal plane defined by the marker placement over the spinous processes. The change in orientation between the lines interconnecting the adjacent markers was used to define each spinal region curvature in degrees. This was calculated by summing all angular changes within each region: lower lumbar (S1L3); upper lumbar (L3-T12); lower thoracic (T12-T6); and upper thoracic (T6-C7) (Fig. 3). The mid-point spinal

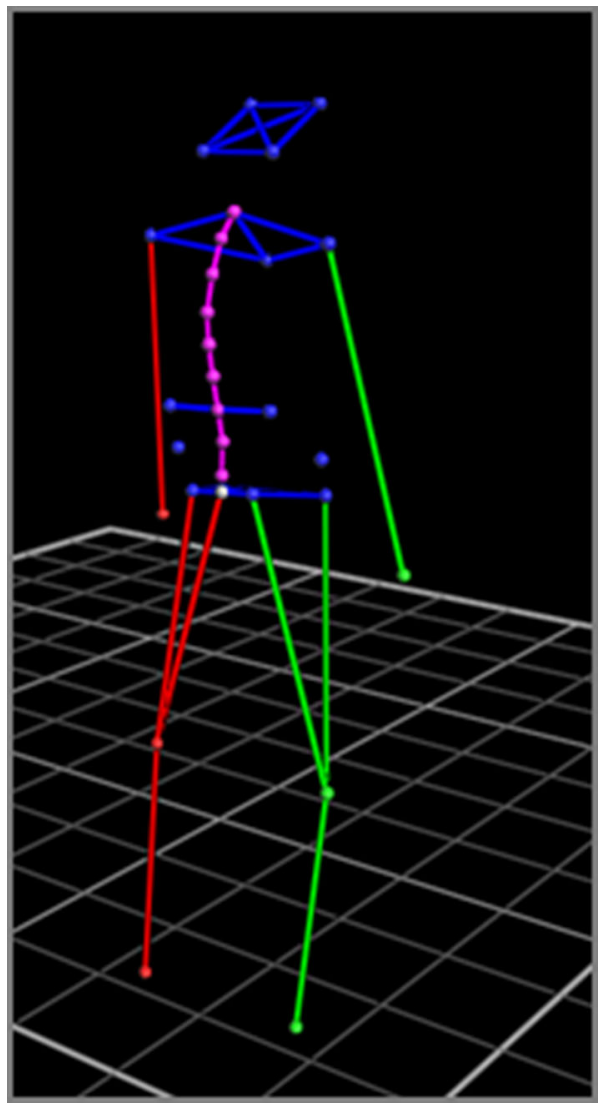

Fig. 2 Labelled marker set as visualised in Vicon ${ }^{\circledR}$

curvature of the subjects' total range of movement was determined for each task. This was calculated as follows: (maximum flexion sagittal spinal angle + maximum extension sagittal spinal angle)/2.

\section{Statistical analysis}

Statistical tests were performed based on the normal distribution and homogeneity of variance [18]. Differences in 
Fig. 3 Sub-division of spinal curvature

baseline characteristics between groups were determined using: one-way ANOVA with post-hoc comparisons (Bonferroni) for age and height; independent samples Kruskal-Wallis for mass and BMI, $\chi^{2}$ for gender; independent $t$ tests for ODQ, VAS, and TSK; and MannWhitney $U$ for DRAM. Following a repeated measures ANOVA for overall group effect, one-way ANOVAs with post-hoc Bonferroni testing determined between group kinematic differences. All statistical testing was performed in SPSS (version 20.0, IBM Corp, Armonk, NY, USA). The alpha level was set at 0.05 [18, 19].

\section{Results}

Fifty NSCLBP subjects (23 AEP, 27 FP) and 28 healthy individuals were included in the final kinematic analysis. One FP participant failed to complete the patient reported measures; therefore, 26 FP patient reported measures data sets were included. Table 2 presents the subject characteristics and patient reported measures. There were no significant differences between groups for age or BMI. Significant differences were observed between groups for gender, mass (AEP vs. FP), and height (AEP vs. FP, FP vs. healthy). The location of back pain was similarly reported between groups with the majority of subjects in both groups reporting central symptoms.

\section{Reliability}

Test re-test reliability was established in a sub-sample of ten healthy volunteers (5 male, 5 female) across four consecutive trials of each functional task. Intraclass correlation coefficients (ICC) were calculated using a two-way mixed model (single measures) with consistency and demonstrated substantial to excellent reliability (ICC 0.746-0.977) across all spinal regions and tasks [20].

\section{Patient reported measures}

Independent $t$ tests for ODQ, VAS, and TSK revealed no significant between differences between the AEP and FP groups indicating comparable baseline levels of disability, pain, and fear of movement between groups. A MannWhitney $U$ test revealed the AEP group to display a significantly more distressed profile compared to the FP group $(p=0.027)$.

\section{Spinal kinematics in the FP, AEP, and healthy groups}

The repeated measures ANOVA for overall group effect was significant $(p=0.014)$ which led to the further exploration of differences within the spinal regions (Tables 3, 4).

Analyses identified differences primarily in the lower thoracic and upper lumbar spinal regions between the FP and AEP group and the FP and healthy group. In both instances, the FP group consistently operated in greater thoraco-lumbar spinal flexion. This was evident between the AEP and FP groups in the upper lumbar and lower thoracic region during all tasks, with the exception of the lower thoracic region during the reach up task $(p=0.103)$. Significant differences were observed between the FP and healthy groups in the lower thoracic region during the stand-to-sit and sit-to-stand tasks and during bending (and returning from) retrieving a pen from the floor. No significant differences were observed between the AEP and healthy groups in any spinal region. No between group significant differences were observed in the upper thoracic or lower lumbar regions during any task.

\section{Discussion}

This is the first study to identify distinct subgroup differences in lower thoracic spinal kinematics during functional activity which can aid in informing personalised rehabilitation strategies for CB-CFT interventions [11].

In-line with recent observations of lumbar movement in LBP subgroups [21], consistent patterns of different spinal movement in the thoraco-lumbar spine were noted between the NSCLBP subgroups throughout the functional tasks. In addition, this region is able to discriminate between the FP and healthy groups, highlighting that distinct increases in thoraco-lumbar flexion in the FP group. These findings support those of Dankaerts et al. [7], where differences were observed in usual sitting upper lumbar posture 
Table 2 Subject baseline characteristics across groups

\begin{tabular}{|c|c|c|c|c|c|}
\hline Variable & $\operatorname{AEP}(n=23)$ & $\mathrm{FP}(n=27)$ & Healthy $(n=28)$ & \multicolumn{2}{|c|}{ Significance } \\
\hline \multicolumn{6}{|c|}{ Subject demographics } \\
\hline \multicolumn{6}{|l|}{ Gender } \\
\hline Males & $4(17.4 \%)$ & $21(77.8 \%)$ & $12(42.9 \%)$ & \multicolumn{2}{|c|}{$p<0.001 *$} \\
\hline Females & $19(82.6 \%)$ & $6(22.2 \%)$ & $16(57.1 \%)$ & & \\
\hline Age (years) & $43.7(11.2)$ & $41.0(10.0)$ & $38.5(11.2)$ & \multicolumn{2}{|c|}{$p=0.238$} \\
\hline Mass (kg) & $68.9(18.0)$ & $82.5(14.6)$ & $72.9(15.2)$ & \multicolumn{2}{|c|}{$p=0.005^{*}($ AEP vs. FP $)$} \\
\hline Height (cm) & $164.9(10.2)$ & $175.9(8.7)$ & $169.4(7.3)$ & \multicolumn{2}{|c|}{$p<0.001 *($ AEP vs. FP/FP vs. $\mathrm{H})$} \\
\hline BMI $\left(\mathrm{kg} / \mathrm{m}^{2}\right)$ & $20.8(4.9)$ & $23.4(3.5)$ & $21.5(4.1)$ & \multicolumn{2}{|c|}{$p=0.127$} \\
\hline \multicolumn{6}{|l|}{ Pain } \\
\hline \multicolumn{6}{|c|}{ Site of back pain, $n(\%)$} \\
\hline Right & $8(34.8 \%)$ & $5(18.5 \%)$ & \multirow[t]{3}{*}{-} & \multirow{3}{*}{\multicolumn{2}{|c|}{-}} \\
\hline Left & $2(8.7 \%)$ & $3(11.1 \%)$ & & & \\
\hline Central & $13(56.4 \%)$ & $19(70.4 \%)$ & & & \\
\hline \multicolumn{6}{|c|}{ Time since pain onset, $n(\%)$} \\
\hline $3-6$ months & $2(8.7 \%)$ & $8(29.6 \%)$ & - & \multicolumn{2}{|l|}{-} \\
\hline 6-12 months & $7(30.4 \%)$ & $2(7.4 \%)$ & - & \multicolumn{2}{|l|}{-} \\
\hline $1-2$ years & $1(4.3 \%)$ & $3(11.1 \%)$ & - & \multicolumn{2}{|l|}{-} \\
\hline $2-3$ years & $0(0 \%)$ & $1(3.7 \%)$ & - & \multicolumn{2}{|l|}{-} \\
\hline $3-4$ years & $2(8.7 \%)$ & $2(7.4 \%)$ & - & \multicolumn{2}{|l|}{-} \\
\hline $4-5$ years & $3(13 \%)$ & $3(11.1 \%)$ & - & \multicolumn{2}{|l|}{-} \\
\hline 5-10 years & $3(13 \%)$ & $4(14.8 \%)$ & - & - & \\
\hline $10+$ years & $5(21.7 \%)$ & $4(14.8 \%)$ & - & - & \\
\hline & $\operatorname{AEP}(n=23)$ & $\mathrm{FP}(n=26)$ & $\mathrm{Hea}$ & 28) & Significance \\
\hline Patient report & & & & & \\
\hline ODQ & 22.5 (11.6) & $21.6(10.0)$ & - & & $p=0.773$ \\
\hline DRAM & $29.8(12.5)$ & 22.7 (10.9) & - & & $p=0.027^{*}$ \\
\hline VAS & $4.6(1.4)$ & $4.5(1.4)$ & - & & $p=0.986$ \\
\hline TSK & $37.5(6.8)$ & $37.6(5.3)$ & - & & $p=0.993$ \\
\hline
\end{tabular}

Values are mean $(\mathrm{SD})$ unless otherwise stated

$F P$ flexion pattern motor control impairment, $A E P$ active extension pattern motor control impairment, $H$ healthy, $B M I$ body mass index (mass $(\mathrm{kg}) /$ height $\left.(\mathrm{m})^{2}\right), \mathrm{kg}$ kilograms, $\mathrm{cm}$ centimetres, $O D Q$ Oswestry Disability Questionnaire, DRAM distress and risk assessment method, VAS Visual Analogue Scale, TSK Tampa Scale of Kinesiophobia

* Significant difference $(p<0.05)$

between AEP and FP groups. However, in contrast to the results reported here, Dankaerts et al. [7] observed differences between AEP and healthy individuals in the upper lumbar region, whilst no differences were observed between FP and healthy individuals. However, similarities are noted between the current study and adolescent cohort data, both identifying significant differences in usual sitting in the upper but not lower lumbar spine [9]. Both Dankaerts et al. [7] and Astfalck et al. [9] demonstrate significant differences between all three groups (FP, AEP, and healthy) in upper lumbar posture which were not replicated in this study. Differences in instrumentation (electromagnetic 3Space Fastrak $\left.{ }^{\circledR}\right)$, subject numbers, and mean age (41.1 compared to 36.0 [7] and 15.6 [9] years) may account for some variation in results as well as observation of static postures rather than functional activities.

Overall, AEP individuals operated in significantly greater extension compared to FP during almost all functional tasks in upper lumbar and lower thoracic regions $(p<0.05)$. This may reflect the more extended nature of the task, where ROM in this region appears to be similar across all groups. The between group differences observed in the lower thoracic region are novel findings in this study. A non-significant trend (following adjustment for gender differences) for a reduction in overall trunk curvature in AEP compared to FP individuals has been observed previously in adolescent cohorts [22], suggesting that the AEP group may adopt a less kyphotic trunk posture compared to 
Table 3 Descriptive and inferential results (one-way ANOVA and post-hoc Bonferroni) for the mid-point sagittal thoracic spinal curvatures for each functional task between the active extension pattern, flexion pattern, and healthy groups

\begin{tabular}{|c|c|c|c|c|c|c|}
\hline \multirow[t]{3}{*}{ Task } & \multicolumn{3}{|c|}{ Classification group descriptives $\left(^{\circ}\right)$} & \multirow{2}{*}{\multicolumn{2}{|c|}{ One-way ANOVA }} & \multirow[t]{3}{*}{ Post-hoc Bonferroni $(p<0.05)$} \\
\hline & \multicolumn{3}{|l|}{ Mean (SD) } & & & \\
\hline & $\operatorname{AEP}(n=23)$ & $\mathrm{FP}(n=27)$ & Healthy $(n=28)$ & $F$ & $p$ & \\
\hline \multicolumn{7}{|l|}{ Upper thoracic } \\
\hline Reach up & $27.2(8.2)$ & $25.3(7.8)$ & $27.1(7.6)$ & 0.485 & 0.618 & - \\
\hline Step down & $34.5(8.2)$ & $33.8(7.9)$ & $35.2(6.9)$ & 0.206 & 0.815 & - \\
\hline Step up & $33.1(7.5)$ & $32.1(7.4)$ & $34.1(6.9)$ & 0.521 & 0.596 & - \\
\hline Box replace & $26.8(8.5)$ & $25.4(7.2)$ & $26.6(7.7)$ & 0.238 & 0.789 & - \\
\hline Box lift & $23.3(9.9)$ & $23.9(7.1)$ & $24.0(8.5)$ & 0.049 & 0.952 & - \\
\hline Stand-to-sit & $22.1(8.8)$ & $20.5(6.7)$ & $22.5(7.8)$ & 0.48 & 0.621 & - \\
\hline Sit-to-stand & $20.4(8.7)$ & $18.8(6.2)$ & $20.6(7.4)$ & 0.454 & 0.637 & - \\
\hline Pick up pen (bend down) & $17.2(10.5)$ & $19.2(9.0)$ & $18.8(8.2)$ & 0.296 & 0.745 & - \\
\hline Pick up pen (return) & $14.3(11.5)$ & $15.1(8.5)$ & $15.6(9.1)$ & 0.099 & 0.905 & - \\
\hline \multicolumn{7}{|l|}{ Lower thoracic } \\
\hline Reach up & $4.4(13.1)$ & $11.1(9.2)$ & $6.4(11.4)$ & 2.344 & 0.103 & - \\
\hline Step down & $9.5(13.2)$ & $18.4(9.1)$ & $12.6(10.3)$ & 4.353 & $0.016^{*}$ & AEP vs. FP \\
\hline Step up & $10.0(12.5)$ & $18.0(9.2)$ & $11.8(10.3)$ & 3.967 & $0.023^{*}$ & AEP vs. FP \\
\hline Box replace & $13.0(10.0)$ & $21.7(8.2)$ & $15.5(11.0)$ & 5.231 & $0.007^{*}$ & AEP vs. FP \\
\hline Box lift & $14.1(9.8)$ & $22.4(7.9)$ & $16.7(10.2)$ & 5.144 & $0.008^{*}$ & AEP vs. FP \\
\hline Stand-to-sit & $8.8(11.2)$ & $18.1(8.5)$ & $10.7(10.9)$ & 5.997 & $0.004 *$ & AEP vs. FP/FP vs. H \\
\hline Sit-to-stand & $7.8(11.0)$ & $17.6(8.1)$ & $9.9(11.2)$ & 6.638 & $0.002^{*}$ & AEP vs. FP/FP vs. H \\
\hline Pick up pen (bend down) & $20.4(9.9)$ & $26.4(6.6)$ & $20.4(7.4)$ & 5.027 & $0.009^{*}$ & AEP vs. FP/FP vs. H \\
\hline Pick up pen (return) & $19.0(8.7)$ & $25.0(6.1)$ & $19.3(7.6)$ & 5.478 & $0.006^{*}$ & AEP vs. FP/FP vs. $\mathrm{H}$ \\
\hline
\end{tabular}

Positive values refer to relative spinal flexion, whilst negative values refer to relative spinal extension

$F P$ flexion pattern motor control impairment, $A E P$ active extension pattern motor control impairment, $H$ healthy, $S D$ standard deviation

* Significant difference $(p<0.05)$

the FP group. However the methods utilised to calculate gross trunk angle do not allow for regional analysis.

Interestingly, no significant between group differences was identified between the AEP and healthy groups, contradicting previous work evaluating static postures [7, 10] and intimates that AEP and healthy individuals may utilise similar spinal movement patterns during functional tasks. There are multiple hypotheses for this observation. AEP individuals subjectively report extension-related activities as pain provocative due to a reduced capacity to control extension movement [5]. Therefore, the functional tasks may not have challenged the spine into substantial extension to elicit pain and maladaptive movement control. Patients often report prolonged exposure to static postures such as sitting and standing as provoking pain; therefore, extension may be more apparent in static postures compared to dynamic functional activity. There are also reported difficulties in accurately classifying AEP individuals, with approximately only 50-62\% of AEP subjects correctly classified by experienced clinicians [23], since the AEP group displayed an overall higher risk of distress compared to the FP group other factors, such as maladaptive trunk muscle activity, or psychosocial factors [24], may be greater contributory mechanisms to pain than spinal movement.

\section{Limitations and future work}

The limitations include optoelectronic spinal measurement which allows only for evaluation of general spinal curvature, as opposed to underlying vertebral movement. Radiographic techniques (e.g., video fluoroscopy) are advancing in NSCLBP research [25] and are warranted to understand true vertebral movement during functional activity. Analysis of other NSCLBP MCI subgroups, trunk muscle activity, and psychosocial factors is required to better understand underlying pain mechanisms [24] as spinal movement alone appears insufficient to comprehensively explain subjectively reported pain presentations. Furthermore, explorations of how NSCLBP subgroups operate over prolonged time periods (using continuous postural measurement devices) will aid in understanding 
Table 4 Descriptive and inferential results (one-way ANOVA and post-hoc Bonferroni) for the mid-point sagittal lumbar spinal curvatures for each functional task between the active extension pattern, flexion pattern, and healthy groups

\begin{tabular}{|c|c|c|c|c|c|c|}
\hline \multirow[t]{3}{*}{ Task } & \multicolumn{3}{|c|}{ Classification group descriptives $\left({ }^{\circ}\right)$} & \multirow{2}{*}{\multicolumn{2}{|c|}{ One-way ANOVA }} & \multirow[t]{3}{*}{ Post-hoc Bonferroni $(p<0.05)$} \\
\hline & \multicolumn{3}{|l|}{ Mean (SD) } & & & \\
\hline & $\operatorname{AEP}(n=23)$ & $\mathrm{FP}(n=27)$ & Healthy $(n=28)$ & $F$ & $p$ & \\
\hline \multicolumn{7}{|l|}{ Upper lumbar } \\
\hline Reach up & $-19.2(12.0)$ & $-11.0(10.0)$ & $-17.4(8.0)$ & 4.824 & $0.011^{*}$ & AEP vs. FP \\
\hline Step down & $-18.0(11.8)$ & $-8.1(9.5)$ & $-15.1(8.4)$ & 6.902 & $0.002^{*}$ & AEP vs. FP/FP vs. H \\
\hline Step up & $-17.0(11.2)$ & $-7.3(8.9)$ & $-14.1(7.8)$ & 7.432 & $0.001^{*}$ & AEP vs. FP/FP vs. H \\
\hline Box replace & $-13.8(10.9)$ & $-3.8(8.6)$ & $-10.1(7.4)$ & 7.844 & $0.001^{*}$ & AEP vs. FP/FP vs. H \\
\hline Box lift & $-11.6(9.4)$ & $-2.4(9.4)$ & $-7.5(7.5)$ & 6.849 & $0.002^{*}$ & AEP vs. FP \\
\hline Stand-to-sit & $-11.9(9.7)$ & $-0.8(8.5)$ & $-6.3(7.3)$ & 10.53 & $<0.001^{*}$ & AEP vs. FP \\
\hline Sit-to-stand & $-10.6(8.7)$ & $-0.6(8.3)$ & $-5.4(7.6)$ & 9.05 & $<0.001^{*}$ & AEP vs. FP \\
\hline Pick up pen (bend down) & $-1.6(7.0)$ & $4.3(6.3)$ & $0.1(5.3)$ & 5.83 & $0.005^{*}$ & AEP vs. FP/FP vs. H \\
\hline Pick up pen (return) & $-1.9(7.3)$ & $3.9(6.2)$ & $1.1(5.4)$ & 4.978 & $0.009^{*}$ & AEP vs. FP \\
\hline \multicolumn{7}{|l|}{ Lower lumbar } \\
\hline Reach up & $-23.3(19.8)$ & $-29.9(18.5)$ & $-22.6(13.9)$ & 1.426 & 0.247 & - \\
\hline Step down & $-21.1(20.8)$ & $-23.7(16.1)$ & $-20.2(9.9)$ & 0.341 & 0.712 & - \\
\hline Step up & $-19.0(19.6)$ & $-22.8(15.7)$ & $-17.4(9.9)$ & 0.922 & 0.402 & - \\
\hline Box replace & $-20.5(17.3)$ & $-24.7(13.5)$ & $-18.9(10.1)$ & 1.258 & 0.29 & - \\
\hline Box lift & $-14.8(16.7)$ & $-20.4(13.7)$ & $-15.0(9.7)$ & 1.426 & 0.247 & - \\
\hline Stand-to-sit & $-11.6(15.0)$ & $-12.0(11.6)$ & $-9.7(9.4)$ & 0.292 & 0.748 & - \\
\hline Sit-to-stand & $-11.0(15.8)$ & $-11.3(12.0)$ & $-9.0(8.9)$ & 0.283 & 0.755 & - \\
\hline Pick up pen (bend down) & $-5.2(16.3)$ & $-5.4(11.9)$ & $-2.2(10.3)$ & 0.506 & 0.605 & - \\
\hline Pick up pen (return) & $-5.3(15.2)$ & $-4.8(13.1)$ & $-2.5(10.0)$ & 0.36 & 0.699 & - \\
\hline
\end{tabular}

Positive values refer to relative spinal flexion, whilst negative values refer to relative spinal extension

FP flexion pattern motor control impairment, $A E P$ active extension pattern motor control impairment, $H$ healthy, $S D$ standard deviation

* Significant difference $(p<0.05)$

habitual spinal movement behaviours, carry-over between therapeutic sessions and longer term movement behaviour change. An important consideration is gender as a confounding factor (FP $77.8 \%$ male, AEP $82.6 \%$ female). Although this is reflective of previous MCI sub-grouped cohorts [7, 9], within-group gender distribution should be considered in future work evaluating the MDCS.

\section{Clinical implications}

The results support the MDCS as a clinical framework for subgrouping NSCLBP, and can inform the refinement of CB-CFT interventions by identifying key regions of differentiated spinal movement. There is strong evidence that individuals with NSCLBP MCI adopt thoraco-lumbar spinal movement behaviours which are consistent with the direction of subjectively reported patterns of pain provocation. Therefore, it is recommended that physical examination incorporates assessment of functional thoracolumbar spine movement, with treatment strategies incorporating targeted functional thoraco-lumbar movement re-education for FP individuals.

\section{Conclusions}

Regional spinal curvatures appear to distinctly differ in MCI subgroups, with the thoraco-lumbar region discriminating between FP and AEP, and FP and healthy groups during functional tasks. The FP group demonstrated more kyphotic postures in these spinal regions, whereas no significant differences between the AEP and healthy groups were observed, suggesting that these groups adopt more similar functional movement strategies. Alternatively, postures or activities may have needed to be more involved or sustained to elicit a response in these groups. The differences observed in the thoracic spine also highlight the importance of incorporating thoracic spine evaluation into NSCLBP assessment. Gender should also be considered as a confounding factor in future studies. 
Acknowledgements Support for the study is acknowledged from Health and Care Research Wales [formerly National Institute of Social, Health and Care (NISCHR) Wales] who provided research officers to support data collection.

\section{Compliance with ethical standards}

Conflict of interest Dr. Rebecca Hemming received funding from Arthritis Research UK as part of the Arthritis Research UK Biomechanics and Bioengineering Centre, Cardiff University. Dr. Rebecca Hemming also received funding via a Presidents Scholarship Award, Cardiff University. For the remaining authors, none were declared.

Ethical approval All procedures performed were in accordance with the ethical standards of the institutional research committee and with the 1964 Helsinki declaration and its later amendments or comparable ethical standards.

Open Access This article is distributed under the terms of the Creative Commons Attribution 4.0 International License (http://crea tivecommons.org/licenses/by/4.0/), which permits unrestricted use, distribution, and reproduction in any medium, provided you give appropriate credit to the original author(s) and the source, provide a link to the Creative Commons license, and indicate if changes were made.

\section{References}

1. Balagué F, Mannion AF, Pellisé F, Cedraschi C (2012) Nonspecific low back pain. Lancet 379(9814):482-491

2. Foster NE, Hill JC, O'Sullivan P, Hancock M (2013) Stratified models of care. Best Pract Res Clin Rheumatol 27(5):649-661

3. Patel S, Friede T, Froud R, Evans DW, Underwood M (2013) Systematic review of randomized controlled trials of clinical prediction rules for physical therapy in low back pain. Spine 38(9):762-769. doi:10.1097/BRS.0b013e31827b158f

4. Foster NE, Hill JC, Hay EM (2011) Subgrouping patients with low back pain in primary care: are we getting any better at it? Man Ther 16(1):3-8. doi:10.1016/j.math.2010.05.013

5. O'Sullivan P (2005) Diagnosis and classification of chronic low back pain disorders: maladaptive movement and motor control impairments as underlying mechanism. Man Ther 10:242-255

6. O'Sullivan P (2004) Clinical instability of the lumbar spine. In: Boyling JD, Jull GA (eds) Grieve's modern manual therapy: the vertebral column, 3rd edn. Elsevier, Amsterdam

7. Dankaerts W, O'Sullivan P, Burnett A, Straker L (2006) Differences in sitting postures are associated with nonspecific chronic low back pain disorders when patients are subclassified. Spine 31:698-704

8. Dankaerts W, O’Sullivan P, Burnett A, Straker L (2006) Altered patterns of superficial trunk muscle activation during sitting in nonspecific chronic low back pain patients: importance of subclassification. Spine 31(17):2017-2023. doi:10.1097/01.brs. 0000228728.11076 .82
9. Astfalck RG, O'Sullivan PB, Straker LM, Smith AJ, Burnett A, Caneiro JP, Dankaerts W (2010) Sitting postures and trunk muscle activity in adolescents with and without nonspecific chronic low back pain: an analysis based on subclassification. Spine 35(14):1387-1395

10. Sheeran L, Sparkes V, Caterson B, Busse-Morris M, van Deursen R (2012) Spinal position sense and trunk muscle activity during sitting and standing in nonspecific chronic low back pain: classification analysis. Spine 37(8):E486-E495

11. Fersum KV, O'Sullivan P, Skouen JS, Smith A, Kvale A (2013) Efficacy of classification-based cognitive functional therapy in patients with non-specific chronic low back pain: a randomized controlled trial. Eur J Pain 17(6):916-928. doi:10.1002/j.15322149.2012.00252.x

12. O'Sullivan P (2000) Lumbar segmental 'instability': clinical presentation and specific stabilising exercise management. Man Ther 5:2-12

13. Von Korff M, Deyo RA, Cherkin D, Barlow W (1993) Back pain in primary care: outcomes at 1 year. Spine $18(7): 855-862$

14. Fairbank JC, Couper J, Davies J, O'Brien J (1980) The Oswestry low back pain disability questionnaire. Physiotherapy 66:271-273

15. Main CJ, Wood PL, Hollis S, Spanswick CC, Waddell G (1992) The distress and risk assessment method. A simple patient classification to identify distress and evaluate the risk of poor outcome. Spine 17(1):42-52

16. Miller RP, Kori SH, Todd DD (1991) The Tampa Scale: a measure of kinesiophobia. Unpublished Report, Tampa

17. Simmonds MJ, Kumar S (1993) Health care ergonomics. Part II: location of body structures by palpation - a reliability study. Int $\mathrm{J}$ Ind Ergon 11(2):145-151. doi:10.1016/0169-8141(93)90008-2

18. Field A (2009) Discovering statistics using SPSS. Sage, London

19. IBM (2012) The calculation of Bonferroni-adjusted $p$-values. IBM. http://www-01.ibm.com/support/docview.wss?uid= swg21476685. Accessed 05 Jan 2015

20. Landis JR, Koch GG (1977) The measurement of observer agreement for categorical data. Biometrics 33(1):159-174

21. Marich AV, Hwang C-T, Salsich GB, Lang CE, Van Dillen LR (2017) Consistency of a lumbar movement pattern across functional activities in people with low back pain. Clin Biomech 44:45-51. doi:10.1016/j.clinbiomech.2017.03.004

22. Astfalck RG, O'Sullivan PB, Straker LM, Smith AJ (2010) A detailed characterisation of pain, disability, physical and psychological features of a small group of adolescents with nonspecific chronic low back pain. Man Ther 15(3):240-247. doi:10. 1016/j.math.2009.12.007

23. Fersum KV, O'Sullivan PB, Kvale A, Skouen JS (2009) Interexaminer reliability of a classification system for patients with non-specific low back pain. Man Ther 14(5):555-561

24. Linton SJ (2000) A review of psychological risk factors in back and neck pain. Spine 25(9):1148-1156

25. Breen A, Breen A (2017) Uneven intervertebral motion sharing is related to disc degeneration and is greater in patients with chronic, non-specific low back pain: an in vivo, cross-sectional cohort comparison of intervertebral dynamics using quantitative fluoroscopy. Eur Spine J. doi:10.1007/s00586-017-5155-y 